\title{
Constitutive Elements of Non-Abelian Gauge Theories
}

\author{
Ademir E. Santana1, Samuel Simon ${ }^{2}$ \\ ${ }^{1}$ International Center for Condensed Matter Physics, Instituto de Fisica, Universidade de Brasilia, Brasilia, Brazil \\ ${ }^{2}$ Deaprtamento de Filosofia, Universidade de Brasilia, Brasilia, Brazil \\ Email: a.berti.santana@gmail.com, samuell@unb.br
}

Received 29 December 2014; accepted 19 January 2015; published 22 January 2015

Copyright (C) 2015 by authors and Scientific Research Publishing Inc.

This work is licensed under the Creative Commons Attribution International License (CC BY).

http://creativecommons.org/licenses/by/4.0/

(c) (i) Open Access

\section{Abstract}

A set, $S$, of constitutive elements characterizing mechanical theories is defined. In $S$, the role played by concepts such as mass, particle, fields and symmetry is discussed. This structure is first used to consider the Nöther's theorem from an algebraic point of view. As examples, we explore non-relativistic quantum mechanics and special relativistic particles. The set $S$ is then applied to analyze non-abelian gauge theories, considering the Higgs mechanism for generation of mass.

\section{Keywords}

\section{Gauge Fields, Constitutive Elements, Lie Symmetry}

\section{Introduction}

With the emergence of subatomic theories, in the 1920s, the problem of establishing the basis of quantum mechanics, considering the classical mechanics counterpart, came about [1] [2]. Attempts to address this problem gave rise, over the decades, to numerous works, following different mathematical approaches and physical motivations. Although much of these investigations have been initially restricted to the analysis of classical and quantum premises in the non-relativistic realm, they have led to important discoveries, such as the notion of entanglement and teleportation, crucial keys for quantum computers and quantum network [3]-[5]; concepts that have been explored in high energy physics [6]. These investigations are mainly considered in two directions, that are at some extent complementary to each other.

One of them is the stochastic methods, that have been used to derive quantum mechanics starting, for instance, from the Liouville equation or from the Fokker-Planck equation [7]-[15]. In another direction, there are attempts exploring the notion of symmetry and representation theories [16]-[20]. The former direction usually emphasizes the nature of the state, being interesting for deriving, for example, the Schrödinger equation, while the 
later, guided by algebraic structures and symmetries, is useful for generalizations, and can accommodate an abroad class of mechanical systems, that include relativistic, non-relativistic and thermal systems [21] [22].

For the case of non-relativistic quantum mechanics, Levi-Leblond [23]-[25] was the first to present a systematic study of unitary representations of the Galilei group, leading to the Schrödinger equation and PauliSchrödinger equation, describing, respectively, spin- 0 and spin 1/2 non-relativistic particles. A consequence, in terms of premises, was that the spin of a particle should be fully described and physically interpreted in terms of the rotation symmetry. It is important to note that, before these works, it was usual to consider spin in the nonrelativistic quantum mechanics as a relativistic remnant of the Dirac equation.

Although representations of Lie group are key aspects to deriving physical theories, this method, as well as the stochastic analysis, has been only partially explored to address the premises of quantum field theories in comparison with other mechanical theories [6] [15] [18] [26]. This is a demanding problem, since new phenomena and concepts need to be analyzed in detail. The situation is more appealing in non-abelian gauge field theories, as the standard model for particle physics, where the nature of the mechanism for the origin of mass is only partially explained through the introduction of the Higgs bosons, presently under experimental test.

In the present work, our main goal is to construct a systematization for mechanical formalisms, which is established by six constitutive elements. In this context, gauge fields are considered by taking into account counterparts of other theories of motion, such as quantum mechanics and one-particle special relativity. The general algebraic structure is supported by physical (experimental) conditions. A first result is a derivation of a Lie-algebra structure associated to the six constitutive elements. This aspect, which is in turn connected to the Nöther theorem, is important to establish the consistency of the number of six constitutive elements. After analyzing the structure of quantum mechanics and special relativity, we investigate non-abelian fields, discussing the concept of mass, from Newton up to Higgs. We have to emphasize that what is new in the present work is the structure of six constitutive elements fixing the content of theories of motion. This aspect is useful, as aforementioned, for the comparative analysis of theories. In this realm, for instance, a fundamental difference between classical and quantum mechanics is not the nature of the Hilbert space, but the experimental condition imposed by the Heisemberg relations.

The paper is organized in the following way. In Section 2, we present the constitutive elements of a mechanics. In Section 3, there is a demonstration that the constitutive elements induce an algebraic structure of Lie algebra in association with the Nöther theorem. In Section 4, the premisses of the special relativity theory and quantum mechanics are analyzed. In Section 5, non-abelian gauge theory is discussed as a mechanical theory. In Section 6, the notion of mass is analyzed. The final concluding remarks are presented in Section 7. In the Appendix, we review some well know aspects of gauge theories in order to make clear the origin of the six constitutive elements.

\section{Constitutive Elements for Theories of Motion}

A theory of movement, a mechanics, can be defined by the following set, $S$, of constitutive elements (CE).

CE1. Reference systems. A reference system is defined from points in the $\mathbb{R}^{4}=\mathbb{R}^{1} \times \mathbb{R}^{3}$, where $\mathbb{R}^{1}$ stands for the time and $\mathbb{R}^{3}$ is the Euclidian space. Time is defined by clocks and the Euclidian space is defined by the constructions of rods. In both the cases, the definitions are given by considering events relative to each other. The mechanical characterizations of the manifold $\mathbb{R}^{4}$ is specified by the kinematical symmetry of the space time. Since a general symmetry is a continuous mapping $\Lambda: \mathbb{R}^{4} \rightarrow \mathbb{R}^{4}, \Lambda$ can be taken as a Lie-group (see next section for a general explanation). It is important to mention that a clock is defined by any periodic system, such that this periodicity depends on the experimental precision.

CE2. Kinematical variables. The set of kinematical variables, $\mathcal{V}=\{A, B, \cdots\}$, is defined as an associative algebra, such that each element of $\mathcal{V}$ is in correspondence with aspects of motion taking place in $\mathbb{R}^{4}$. There is a subset $\mathcal{V}_{C} \subset \mathcal{V}, \mathcal{V}_{C}=\left\{\xi_{1}, \xi_{2}, \cdots, \xi_{r}\right\}$ from which all the elements of $\mathcal{V}$ are constructed in the form

$$
A=\sum_{n_{1} n_{2} \cdots n_{r}=0}^{\infty} a_{n_{1} n_{2} \cdots n_{r}}\left(\xi_{1}\right)^{n_{1}}\left(\xi_{2}\right)^{n_{2}} \cdots\left(\xi_{r}\right)^{n_{r}}
$$

The set $\mathcal{V}_{C}$ will be called the set of canonical variables.

CE3. Mechanical system. A mechanical system is defined as the object under movement. It can be classified by two categories of primitive concepts. One is the material point, specified by a set of local points of $\mathbb{R}^{4}$ and 
endowed with the content of momentum. The other is the concept of a field, which is a mechanical system with no local characteristics in $\mathbb{R}^{4}$ and not reducible to material point; but in general it is endowed with the content of momentum [27] [28]. Mechanical systems, described by points or by field, or both, can interact with each other. The definition of momentum will be given later in association with the specification of a mechanical system (see CE6).

CE4. State of a mechanical system. The mechanical state $\mathcal{E}$ is defined by a set of kinematical variables, expressing the maximal mechanical information that can be obtained from a system. Considering the pair $(\mathcal{E}, A)$, defined by the state of a mechanical system and a kinematical variable $A \in \mathcal{V}$, there is a mapping $\mathcal{M}:(\mathcal{E}, A) \rightarrow \mathbb{R}^{n}$, such that $\mathcal{M}$ is associated with the measurement of $A$. A kinematical variable with this type of association is called an observable. There is another set of variables that describes the generators of changes of the state associated with the motion. In some cases the set of observables and the set of generator of changes are identical to each other, but this is not the case in general. The mechanical state defines a system in motion from a mechanical point of view.

CE5. Changes in the state of mechanical system. The changes in the state $\mathcal{E}$ are defined by changes in the canonical variables. Consider that $\mathcal{E}$ is a function of, at least, part of the canonical variables, say $\mathcal{E}\left(\xi_{1}, \xi_{2}, \cdots, \xi_{n}\right)$, with $n \leq r$, then there is a mapping $\Omega: \mathcal{E}\left(\xi_{1}, \xi_{2}, \cdots, \xi_{n}\right) \rightarrow \mathcal{E}\left(\xi_{1}^{\prime}, \xi_{2}^{\prime}, \cdots, \xi_{r}^{\prime}\right)$ which is defined by local laws, leading to a description of the mechanical system based on differential equations. Considering local laws, in the next section, we discuss the nature of Lie group of transformations $\Omega$. Therefore, $\Omega$ has to be compatible with the transformations $\Lambda$. One specific case is when $\Omega \equiv \Lambda$; however, $\Lambda$, the space-time symmetries, can be only a sub set of transformations $\Omega$. This is the case of gauge theories. In addition, as a physical imposition, $\mathcal{M}$ is invariant by the transformations $\Omega$; this means that one can discuss the motion of a system, considering a change in the state, or a change in the observables, or both. These three possibilities, however, have to be compatible to one another. An example of such possibilities is quantum mechanics, which can be built up in the Schrödinger or Heisenberg or Dirac picture. It is important to emphasize that gauge conditions, introduced by symmetries, determine the way the interaction takes place in a system. Therefore, changes in a mechanical state is defined by the symmetry $\Omega$, giving rise to causal relations among two different conditions of the state of the system. For an infinitesimal association between $\mathcal{E}=\mathcal{E}\left(\xi_{1}, \xi_{2}, \cdots, \xi_{n}\right)$ and $\mathcal{E}^{\prime}=\mathcal{E}\left(\xi_{1}^{\prime}, \xi_{2}^{\prime}, \cdots, \xi_{r}^{\prime}\right)$, we have an differential equation invariant under $\Omega$. The Lie symmetry structure of $\Omega$ (next section) is a central element to define the physical conditions of causality [29] [30]

CE6. Specification of mechanical systems. The specification of a particular mechanical system is given by a function of the state of the system, $\mathcal{L}(\mathcal{E})$, which is an invariant under $\Omega$. The state function $\mathcal{L}(\mathcal{E})$ is called the Lagrangian density and is used to get the causal law among states, by a variational principle, defined by the action defined by

$$
\mathcal{A}=\int \mathrm{d} x^{4} \mathcal{L}(\mathcal{E})
$$

The equations of motion, the causal law, will be the Euler-Lagrange equations and are given by the functional equation $\delta \mathcal{A}=0$. (We do not consider here no-local causal law, leading a description of systems based on integral-differential equations.)

\section{Lie-Algebra Structure of $\Omega$ and Nöther's Theorem}

In this section, using physical (experimental) ingredients of the motion, we show that the set of transformations $\Omega$ is equipped with a Lie-algebra structure. We consider a Heisenberg description, where the mechanical changes are determined by the kinematical variables of the linear space $\mathcal{V}=\{A, B, \cdots\}$ (the state $\mathcal{E}$ is fixed). The change in an element $A \in \mathcal{V}$ is described by a parameter $\tau \in \mathbb{R}$, such that $\delta A=A(\tau+\delta \tau)-A(\tau)$. On the other hand, this change can be specified by the action of a generator, say $B \in \mathcal{V}$, using a linear mapping $():, \mathcal{V} \times \mathcal{V} \rightarrow \mathcal{V}$, such that $\delta A=(A, B) \delta \tau$. This mapping (, ) equips the vector space $\mathcal{V}$ with an algebraic structure, that remains to be identified [31].

For simplicity, we consider that each element of $\mathcal{V}$ can be interpreted as a physical observable and a generator of changes. Then a physical restriction is that one variable $C$ cannot impose mechanical changes on itself; this means that $(C, C)=0$. Taking $C=A+B$, then we have

$$
(A, B)=-(B, A)
$$

that is, the mapping (,) is antisymmetric. 
Since $\mathcal{V}$ is an associative algebra, there is a product, $\circ$, defined in $\mathcal{V}, \circ: \mathcal{V} \rightarrow \mathcal{V}$, such that $A \circ B=A B$ $=C \in \mathcal{V}$ for $A, B \in \mathcal{V}$. Then an infinitesimal change in $C$ is written as $\delta C=(\delta A) B+A(\delta B)$. Assuming that this change is carried out by a variable $D$, specified by a parameter $\varsigma$, we have $\delta A=(A, D) \delta \varsigma$ and $\delta B=(B, D) \delta \varsigma$ and $\delta C=(C, D) \delta \varsigma$. Hence we have

$$
(A B, C)=(A, C) B+A(B, C),
$$

which is the derivation of the Leibnitz rule, defining the association between the associative product $\circ$ and the mapping $(,$,$) .$

Let $D \in V$ given by $D(\varsigma)=(A(\varsigma), B(\varsigma))$, such that, after a change $\delta \varsigma$ in the parameter $\varsigma$, one has

$$
D(\varsigma+\delta \varsigma)=(A(\varsigma+\delta \varsigma), B(\varsigma+\delta \varsigma)) .
$$

considering now that $\delta A=(A, C) \delta \varsigma$ and $\delta B=(B, C) \delta \varsigma$ and $\delta D=(D, C) \delta \varsigma$, we derive

$$
(A,(B, C))+(C,(A, B))+(B,(C, A))=0,
$$

which is the Jacobi identity. Then the mapping $($,$) equips the linear space \mathcal{V}$ with a Lie-algebra with derivation.

From these results, we observe that an invariant quantity, say $I(\mathcal{E})$, derived from a parameterized transformation $\Omega$ on the action $\mathcal{A}$, given in Equation (1), is an invariant under a Lie group. In other words, the quantity $I(\mathcal{E})$ is a Lie symmetry invariant; and as such it can be written in association with a conserved current. Therefore, this provides an algebraic way to derive the Nöther's theorem. This structure of Lie symmetry is a basic tool for defining the physical (experimental) notion of causality [29] [30].

\section{Two Examples: Special Relativity and Quantum Mechanics}

In this section we investigate the constitutive elements of mechanical system with two examples: one particle special relativistic system and one particle quantum mechanics.

\subsection{Special Relativity}

The constitutive elements of a particle in special relativity are identified in the following way.

CE1. Reference systems. The reference systems is defined from points in the $\mathbb{R}^{4}=\mathbb{R}^{1} \times \mathbb{R}^{3}$, where $\mathbb{R}^{1}$ stands for the time and $\mathbb{R}^{3}$ is the Euclidian space. Time is defined by clocks and the Euclidian space is defined by the construction of rods. The manifold $\mathbb{R}^{4}$, with 4-vectors written as $x=\left(x^{0}, x^{1}, x^{2}, x^{3}\right)$, is equipped with a Minkowski scalar product, with metric $g^{\mu v}$ defined by $\operatorname{diag}\left(g^{\mu v}\right)=(1,-1,-1,-1)$ (where, $\mu, v=0,1,2,3$ ). The physical kinematical symmetries of the space time is the linear mapping $\Lambda: \mathbb{R}^{4} \rightarrow \mathbb{R}^{4}, \bar{x}^{\mu}=\Lambda^{\mu}{ }_{\nu} x^{\nu}$, such that the scalar product $x \cdot y=g_{\mu v} x^{\mu} y^{v}$ is invariant. The set of transformation $\Lambda^{\mu}{ }_{v}$ is the Lorentz-Lie group. With this structure, the manifold $\mathbb{R}^{4}$ is called the Minkowski space, $\mathbb{M}$.

CE2. Kinematical variables. The set of kinematical variables, $\mathcal{V}=\{A, B, \cdots\}$, is defined by the associative algebra of functions $f: \mathbb{M} \rightarrow \mathbb{M}$, in general of class $C^{n}$, such that $f$ is a scalar, or a 4-vector or a tensor. The subset $\mathcal{V}_{C} \subset \mathcal{V}$, of canonical coordinates are given by the 4-position, $x$, and the 4-momentum, $p$.

CE3. The mechanical system. We consider as a mechanical system a material point. The mass of material point is defined with the characteristics of a Newtonian material point with inertia, but now mass, $m$, has an additional attribute: it is a Lorentz scalar, given by the relation $p \cdot p=g_{\mu v} p^{\mu} p^{v}=m^{2}$ (throughout the paper we consider natural units, with $c=\hbar=1$ ). In this case, $m$ is a consequence of the Lorentz symmetry.

CE4. The state characterizing a material point can be given by $\mathcal{E}=(x, p)$, physically describing the following aspects. a) Location: the 4-vector $x$ specify the location of the material point in space-time. If we have a system with $N$ material points, as a relativistic gas, then we have $N$ points in $\mathbb{M}$ accounting for the distribution of particles in the Euclidian space and its time evolution. b) Movement: the second aspect of the state $\mathcal{E}$, the 4-momentum $p$, stands for an intrinsic characteristic of the system associated with its motion. In this case, it is related to the 4-velocity of the material point. We could include in $\mathcal{E}$ the 4-acceleration and other high derivatives. But the choice is determined by experiments. Considering the pair $(\mathcal{E}, A)$, where $A$ is an observable, the mapping $\mathcal{M}:(\mathcal{E}, A) \rightarrow \mathbb{R}$ is given by the fact that $A$ is defined from $\mathbb{R}$. For instance, if $A$ is a tensor, its entries are real. 
CE5. Changes in the state of mechanical system. The changes in the state given by $\Omega: \mathcal{E}(q, p) \rightarrow \mathcal{E}\left(q^{\prime}, p^{\prime}\right)$ are defined by the Lorentz group, $\Omega \equiv \Lambda$.

CE6. The specification of the particular mechanical system is given by a function of the state of the system, $\mathcal{L}(\mathcal{E})$ which is an invariant under $\Omega$ that is taken here as $\Omega=\Lambda$. The state function, the Lagrangian density, $\mathcal{L}(\mathcal{E})$ is then used to get the causal law among states, by the variational principle, defined by the action $\mathcal{A}=\int \mathrm{d}^{4} x \mathcal{L}(\mathcal{E})$. This leads to the equation of motion of one-particle special relativity.

It is important to consider now another representation for relativistic particles, the Poisson-Liouville formulation of special relativity. In this case, the state is defined by a $\Lambda$-invariant density of probability $f(q, p ; t)$ satisfying the Liouville equation

$$
\partial_{\tau} f(q, p ; t)=p^{\mu} \frac{\partial f}{\partial q^{\mu}}=0,
$$

which can be written as $\left\{p^{2}, f(q, p ; t)\right\}=0$, where

$$
\{A, B\}=\frac{\partial A}{\partial q^{\mu}} \frac{\partial B}{\partial p_{\mu}}-\frac{\partial A}{\partial p^{\mu}} \frac{\partial B}{\partial q_{\mu}}
$$

is the Poisson bracket. In this representation, the generators of Lorentz transformation are given by

$$
\hat{M}^{\mu \nu}=\left\{m^{\mu \nu}, .\right\}
$$

The kinematical variable $m^{\mu v}=q^{\mu} p^{v}-q^{v} p^{\mu}$ is an observable, while the kinematical variable $\hat{M}^{\mu v}$ fullfils the Lie algebra

$$
\left[\hat{M}_{\mu v}, \hat{M}_{\sigma \rho}\right]=-i\left(g_{\mu \rho} \hat{M}_{v \sigma}-g_{v \rho} \hat{M}_{\mu \sigma}+g_{\mu \sigma} \hat{M}_{\rho v}-g_{v \sigma} \hat{M}_{\rho \mu}\right),
$$

Explicitly, we then note a separation of generators of symmetry, as $\hat{M}^{\mu v}$, and observables, as $m^{\mu v}$, defined in the phase space. In this formulation, the mapping $\mathcal{M}:(\mathcal{E}, A) \rightarrow \mathbb{R}^{n}$, defining measurables quantities, is given by

$$
\langle A\rangle=\int \mathrm{d} q \mathrm{~d} p f(q, p ; t) A(q, p) .
$$

For $f(q, p ; t)=\delta(q-q(t)) \delta(p-p(t))$, where $q(t)$ and $p(t)$ are solutions of the relativistic Einstein equations for one-particle, the Poisson-Liouville formulation is equivalent with the Hamilton equations.

The representations of the Lorentz algebra, given in Equation (5) has been analyzed in the literature [21]. However, considering the set of constitutive elements $\mathcal{S}$, we then have other possibilities of representation for the algebra given by Equation (5). One example is given by introducing the following operators in phase space

$$
\hat{P}_{\mu}=i\left\{p_{\mu},\right\}=-i \frac{\partial}{\partial q^{\mu}}, \hat{Q}_{\mu}=i\left\{q_{\mu},\right\}=i \frac{\partial}{\partial p^{\mu}}, \bar{P}_{\mu}=\mathbf{1} \cdot p_{\mu}, \bar{Q}_{\mu}=\mathbf{1} \cdot q_{\mu} .
$$

Then we can define

$$
P_{\mu}=a \bar{P}_{\mu}+b \hat{P}_{\mu} ; Q_{\mu}=c \bar{Q}_{\mu}+d \hat{Q}_{\mu} .
$$

Now we can calculate the values of the constants $a, b, c$ and $d$, leading to a representation of the Poincaré-Lie algebra by introducing the generator

$$
M_{\mu v}=Q_{\mu} P_{v}-Q_{v} P_{\mu} .
$$

In order to get a representation of the Lorentz-Lie algebra, given by Equation (5), the constants $a, b, c$ and $d$ in Equation (6) have to satisfy the following condition $a d+b c=1$. This gives rise to many other different representations to be explored. These representations as well as the non-relativistic limit, leading to the Galilei-Lie algebra, will be analyzed in detail elsewhere. Let us discuss now, a quantum mechanical system.

\subsection{Quantum Mechanics}

A mechanical theory describing the movement of one quantum particle is given by the following set, $S$, of constitutive elements. 
CE1. Reference systems. A reference systems is defined from points such that the mapping $\Lambda: \mathbb{R}^{4} \rightarrow \mathbb{R}^{4}$ is the Galilean group.

CE2. The set of kinematical variables, $\mathcal{V}=\{A, B, \cdots\}$, is defined by the associative algebra of linear operators acting on a Hilbert space, $\mathcal{H}$, defined by the complex functions $f: \mathbb{R}^{4} \rightarrow \mathbb{R}^{4}$, in general of class $C^{n}$, such that $f$ is a scalar, tensor or an spinor in the Euclidian space $\mathbb{R}^{3}$. The subset $\mathcal{V}_{C} \subset \mathcal{V}$, of canonical coordinates as given by the 3-position, $\boldsymbol{q}$, and the 3-momentum, $\boldsymbol{p}$. In a quantum mechanical system, by experimental reasoning, the Heisenberg relations have to be fulfilled. For instance for position and momentum, we have $\left[q^{i}, p^{j}\right]=i \delta^{i j}$.

CE3. The mechanical system is a non-relativistic material point, interacting to each other by a potential. For electrons such a potential is given by the electromagnetic field.

CE4. The state of a quantum mechanical system is $\mathcal{E}=\psi(\boldsymbol{q}, t)=\langle\boldsymbol{q} \mid \psi(t)\rangle$, such that $|\psi(t)\rangle \in \mathcal{H}$ and $\langle\boldsymbol{q}|$, the dual of $|\boldsymbol{q}\rangle$, is an element of a basis expanding the Hilbert space. From a physical point of view, $\psi(\boldsymbol{q}, t)$ describes the following aspects. a) The location of a particle in space and time $(\boldsymbol{q}, t)$ since $\psi(\boldsymbol{q}, t)$ is a amplitude of probability. If we have a system with $N$ material points, as a quantum mechanical gas, then we have $N$ points accounting for the distribution of particles in the Euclidian apace and its time evolution. b) the probability content of $\psi(\boldsymbol{q}, t)$ stands for an intrinsic characteristic of the system associated with the movement. Considering the pair $(\mathcal{E}, A)$, where $A$ is an observable, the mapping $\mathcal{M}:(\mathcal{E}, A) \rightarrow \mathbb{R}$ is given by the bilinear form $\langle A\rangle=\langle\psi(t)|A| \psi(t)\rangle$.

CE5. Changes in the state of mechanical system. The changes in the state $\Omega:|\psi(t)\rangle \rightarrow \mathcal{E}\left(q^{\prime}, p^{\prime}\right)$ are given by the Galilei group [27].

CE6. The specification of the particular mechanical system is given by a function of the state of the system, $\mathcal{L}(\mathcal{E})$ which is an invariant under $\Omega$, the Galilei group. The state function, the Lagrangian density, $\mathcal{L}(\mathcal{E})$ is then used to get the causal law among states, by the variational principle, defined by action by $\mathcal{A}=\int \mathrm{d} x^{3} \mathcal{L}(\mathcal{E})$.

This leads to the Schrödinger equation describing the motion of a quantum particle. In this case the Lagrangian is $\mathcal{L}(\mathcal{E})=\psi^{*}(\boldsymbol{q}, t)\left(i \partial_{t}-H\right) \psi(\boldsymbol{q}, t)$, where $H$ is the Hamiltonian of system.

The representation of quantum mechanics in phase space has been explored starting with the Wigner formalism, based on the notion of quasi-distribution function. In this case representations of the Galilei group in phase space has been analyzed following in parallel to the Lorentz symmetry, considered in the last Section [21]. This aspect will be developed in a separate investigation. However, it is important to note here that different formalisms of classical or quantum mechanics can be accommodated in the set of Constitutive Elements and analyzed in comparison to one another.

\section{Constitutive Elements of Non-Abelian Gauge Fields}

One goal in this section is to consider the Constitutive elements of non-abelian gauge-fields, in order to perform a conceptual analysis, in the next section, about the notion of mass, from Newton to Higgs. In order to fix the notation and to emphasize important aspects of our discussion, we review in the Appendix some elements of a gauge theory.

CE1. Reference systems. In a (abelian or non-abelian) gauge theory, the reference systems are taken from special relativity, i.e. the Minkowski space. The set of transformation $\Lambda^{\mu}{ }_{v}$ is the Lorentz-Lie group and the manifold $\mathbb{R}^{4}$ is Minkowski space, $\mathbb{M}$.

CE2. The set of kinematical variables, $\mathcal{V}=\{A, B, \cdots\}$, is defined by the associative algebra of linear operators acting on a Fock-Hilbert space, $\mathcal{F}$, defined such that $\mathcal{F}=\oplus_{m} \mathcal{H}^{\otimes m}, m=0,1,2, \cdots$. The Heisenberg relations have to be satisfied by such field operators, that for matter fields, say $\hat{\psi}(x)$ and $\hat{\psi}^{\dagger}(x)$, leads to the following relations [27] [28]

$$
\begin{aligned}
& {\left[\hat{\psi}(x ; t), \hat{\psi}^{\dagger}\left(x^{\prime} ; t\right)\right]=\delta\left(x-x^{\prime}\right),} \\
& {\left[\hat{\psi}(x ; t), \hat{\psi}\left(x^{\prime} ; t\right)\right]=\left[\hat{\psi}^{\dagger}(x ; t), \hat{\psi}^{\dagger}\left(x^{\prime} ; t\right)\right]=0 .}
\end{aligned}
$$

The procedure of quantization is carried out, consistently, by the definition of a generating functional for correlation function of the system. With this procedure, the basic physical observables are established. In particular, the cross-section of a physical process, such as the scattering of particles, can be defined and compared with experiments by using correlation functions. 
CE3. The mechanical system. In quantum field theory, a mechanical system is described by a field. A gauge field will describe the process of interaction among the matter field; that is the case of the Dirac field describing electrons or quarks. The notion of mass of a field is still defined with the characteristics of a Newtonian material point with inertia, and is a Lorentz scalar, obtained from the energy-momentum tensor.

CE4. The state characterizing a field is a vector in $\mathcal{F}=\oplus_{m} \mathcal{H}^{\otimes m}$. One important state is the vacuum, the fundamental states $\mathcal{E}=|\Phi\rangle \in \mathcal{F}$, from which we can construct other states describing for instance one-electron, or two-electrons, etc. interacting with each other. It is important to note that the name "one-eletron" is still designated by one-particle state. However, we describe particles here as follows: a sharp-localized field in the Minkowsky space, $\mathbb{M}$. The vacuum state is Lorenz invariant. Other aspects are worth mentioning. a) Location: a 4-vector $q$ that specifies the location of a particle in space-time is an information within the state of the system, say $|\Psi\rangle \in \mathcal{F}$. b) Movement: as in the relativistic particle case, a 4-momentum, $p$, stands for an intrinsic characteristic of the system associated with the motion. Considering the pair $(|\Psi\rangle, A)$, where $A$ is an observable, the mapping $\mathcal{M}:(|\Psi\rangle, A) \rightarrow \mathbb{R}$ is given by the bilinear form $\langle\Psi|A| \Psi\rangle$.

CE5. Changes in the state of the mechanical system. The changes in the state $|\Psi\rangle$ driven by a symmetry $\Omega$ have the Lorentz group as a sub set of symmetries. Usually, the Heisenberg representation is used, such that the states are fixed and the time evolution is driven by the evolution of the fields. The symmetry $\Omega$ includes the gauge symmetry, as specified in the Appendix.

CE6. Mechanical system. The specification of a particular mechanical system is given by the Lagrangian density $\mathcal{L}(\mathcal{E})$, invariant by $\Omega$; i.e.

$$
\mathcal{L}=\bar{\psi}(x)\left[i D_{\mu} \gamma^{\mu}-m\right] \psi(x)-\frac{1}{4} F_{\mu \nu} F^{\mu v}-\frac{1}{2 \alpha}\left(\partial^{\mu} A_{\mu}^{r}(x)\right)^{2}+A_{\mu}^{r}(x) J^{\mu}(x),
$$

where $\psi(x)$ describes the matter field and $A_{\mu}^{r}(x)$ is the gauge field; $F^{\mu v}$ is the tensor field (see the Appendix). This Lagrangian is invariant under transformations of symmetry $\Omega$, which include $\Lambda$, but goes beyond that to consider internal gauge transformation, defined by the adjoint representation of the symmetry group specified by the group structure constants, $c_{r s l}$. Using the variational principle, defined by action by $\mathcal{A}=\int \mathrm{d} x^{4} \mathcal{L}\left(\psi, A_{\mu}^{r}\right)$, we obtain the field equations. The gauge and Lorentz invariant generating functional, $Z[\mathcal{L}]$ leads to perturbative methods to calculate correlation functions. It is important to mention that the correct $Z[\mathcal{L}]$ demands the introduction of two other elements [21]: i) in order to take into account the noncommutativity of the fermion fields, $\psi$ has to be a Grassmann variable; ii) for accounting the the gauge invariance of $Z[\mathcal{L}]$, ghost fields are introduced in the Lagrangian given in Equation (7), following the Fadeev-Popov methods; iii) the gauge field is massless in order to keep the gauge invariance of $\mathcal{L}$. However, to account for mass, as for the case of electro-weak interaction, a boson field is introduced in $\mathcal{L}$, such that methods of spontaneous symmetry breaking can be explored, as a mechanism for giving rise to mass. In the sequence we analyze this aspects in more detail.

\section{The Notion of Mass from Newton to Higgs}

We analyze now the notion of mass. Our aim here is not to provided a complete historical account about the developments of the concept of mass, but describe the basic improvements in the concept of mass considering the set of Constitutive Elements (CEs). We start with the concept of mass as it was first introduced by Newton in his Principia, Book I, Definition I, as the quantity of matter [32]. In the Book I, Definition III, Newton introduce mass also as a measure of the inertia; i.e. the resistance of a particle to have its state of motion changed by the interactions with another particle (taking as an example a mechanical system described by two particles). The quantity of motion, the momentum, is introduced in Definition II. As emphasized first by Poisson, the notion of material point (or material particle) was implicitly given in the Newton's definition of mass. Such a notion was generalized also by Newton, considering problems in hydrodynamics, following the Pascal's achievements. From the XVIII Century on, with Euler, Lagrange, Laplace, Hamilton and Poisson, a new formalism for classical mechanics is constructed, using new concepts such as energy and gravitational potential. Later, the notion of mass was included in concepts such as the energy momentum-tensor, in order to accommodate the mechanical description of the continuum media. All these concepts were then generalized in two aspects: to accommodate relativistic particles and subatomic physics.

Considering the mechanical constitutive elements, the notion of mass arises as an element defining the 
characteristics of a particle, taken as a primitive concept. As such, mass has to be an invariant under the spacetime symmetry $\Lambda$. However, a particle can be characterized by other invariants. Considering for instance an electromagnetic-interacting particles, beyond the mass, the electrical charge is another primitive characteristic, also $\Lambda$-invariant, characterizing a particle such as an electron. In a more general condition, a particle is fully defined by the set of invariants under the more general symmetry $\Omega$. This invariance accommodates internal symmetry, such as spin and color. The procedure of measurement of mass is defined by the process of experimental characterization of the symmetry $\Omega$. In addition, due to the equivalence principle in general relativity, the mass is also considered as the source (the charge) of the gravitational field, being present in the energymomentum tensor, which is used to defining the curvature in space-time, according to the Einstein equations.

Considering the particle-physics standard model, the mass is introduced by the Higgs mechanism of spontaneous symmetry breaking. As we have observed in the previous section, the gauge field is a zero mass-field by the definition of the gauge symmetry. The original Lagrangian can be improved by introducing a Higgs field in interaction with the gauge field, in order to give rise to a mass term, exploring the concept of spontaneous symmetry breaking, in a way which is similar to the Ginzburg-Landau theory for superconductivity. Although this is an intricate and ingenious mechanism, providing to some extent an explanation for the appearance of mass, the primitive kinematical characteristic of mass is still the same. This is due to the dispersion relation of a particle, which associates the notion of mass in quantum field theory with the primitive concept of matter introduced by Newton.

\section{Concluding Remarks}

In this work we have characterized a mechanical theory from the point of view of six Constitutive Elements (CEs), that is: CE1, the reference systems; CE2, the kinematical variables; CE3, the mechanical system; CE 4, the state of a mechanical system; CE5, the changes in the state of a mechanical system; CE6, the specification of a (particular) mechanical system. These CEs are introduced by taking as a starting point the symmetries of space-time, which in turn is associated with measurement procedures. Such a structure gives rise to a unified description for theories of motion and has been used here to analyze the Newtonian mechanics, fluid mechanics, non-relativistic quantum mechanics, one-particle special relativity and quantum field theories.

From this structure the main results include: i) notions such as particle and fields are described under the unified perspective of a mechanical theory; ii) the demonstration that the CEs has a Lie-symmetry structure in association to the Nöther theorem for conservation laws; iii) considering the Dirac theory for relativistic HamiItonian mechanics, we obtain a general family of representation of the Lorentz group in phase-space; (iv) nonabelian gauge fields are taken as a representation of the six CEs and, under this perspective, the notion of mass is then analyzed since Newton—regarded as the quantity of matter and a measure of the inertia—, to Higgsassociated to the notion of spontaneous of symmetry breaking.

Some aspects of this analysis are in order. First we notice that the experimental nature of the movement leads to a specific nature of mechanics. For instance, we conclude that a crucial difference between classical and quantum mechanics is the experimental conditions imposed by the Heisenberg relations. In this case, a mechanical theory for subatomic systems is intrinsically different from a classical mechanics. But how far is one to the other? Indeed, from the perspective of the set of CEs, one would say that the systems are described by the same mechanical theory, where the differences are emerging from the representation. In particular, this implies that there is nothing "intrinsically quantum mechanical" with the Hilbert space. This is the case for classical theories defined in the simplectic Hilbert spaces [21]. Similar achievements are derived from the comparative analysis of a relativistic and a non-relativistic classical particle. Here the experimental condition of invariance of the velocity of light imposes the Lorentz symmetry for the space-time, such that the Galilei group arises from a low velocity limit. From this point of view, keeping in mind the set of CEs, we conclude that the space and time symmetries (the kinematics) used for describing a specific movement is strongly associated with our experimental capacity. In other words, depending on the prescription, we can use different kinematics, which are in turn defined by measurements. That means that, notions like space and time are fully specified in physics by the relations among objects in movement, which is the main characteristic used for defining a measurement process. This leads us to the conclusion that the Galilei or the Lorentz symmetries are constrained by the experimental conditions; and as such, these set of symmetries are not ontological structure of the space time. This is the case of the conformal symmetry, that can be broken by the Higgs-like mechanism. Therefore, considering the set of 
CEs, a theory is valid by itself in a domain defined by the experimental characterization of the movement. This establishes a picture of relations among theories, that combines symmetry and representations. Discarding experimental evidences, one can extrapolate such a picture in different directions, which can be mathematically consistent, but can no longer be called a mechanical theory.

Finally, it is important to mention that, the structure of the CEs can be extended to equilibrium thermodynamics and thermal quantum field theories, by using thermofield dynamics [21] [33]. This analysis is in progress and will be presented elsewhere.

\section{Acknowledgements}

The authors thank F. C. Khanna, for the discussions and for his interest in this work, and CNPq of Brazil, for financial support.

\section{References}

[1] Jammer, M. (1954) Concepts of Space. Dover, N. York.

[2] Jammer, M. (1974) The Philosophy of Quantum Merchanics: The Interpretations of QM in historIcal Perspective. John Wiley and Sons, N. York.

[3] Brida, G., et al. (2011) Foundations of Physics, 41, 305. http://dx.doi.org/10.1007/s10701-009-9396-4

[4] Omnès, R. (2013) Foundations of Physics, 43, 1339. http://dx.doi.org/10.1007/s10701-013-9750-4

[5] Fox, M. (2006) Quantum Optics, an Introduction. Oxford University Press, Oxford.

[6] Di Domenico, A., et al. (2012) Foundations of Physics, 42, 778. http://dx.doi.org/10.1007/s10701-011-9575-y

[7] Brooke, J.A. (1984) International Journal of Theoretical Physics, 23, 783. http://dx.doi.org/10.1007/BF02214066

[8] Halabi, T. (2013) Foundations of Physics, 43, 1252. http://dx.doi.org/10.1007/s10701-013-9743-3

[9] Khrennikov, A. (2010) Foundations of Physics, 40, 1051. http://dx.doi.org/10.1007/s10701-009-9392-8

[10] Olavo, L.S.F. (2004) Foundations of Physics, 34, 891. http://dx.doi.org/10.1007/s10701-009-9392-8

[11] Olavo, L.S.F. (2000) Physical Review A, 61, Article ID: 052109. http://dx.doi.org/10.1103/PhysRevA.61.052109

[12] Olavo, L.S.F., Lapas, L.C. and Figueiredo, A.D. (2012) Annals of Physics, 327, 1391-1407. http://dx.doi.org/10.1016/j.aop.2012.01.004

[13] Olavo, L.S.F. (2014) Quantum Mechanics: Principles, New Perspectives, Extensions and Interpretations. Noca Science, New York.

[14] Smolin, L. (2012) Foundations of Physics, 42, 1239-1261. http://dx.doi.org/10.1007/s10701-012-9666-4

[15] Prugovečki, E. (1984) Stochastic Quantum Mechanics and Quantum Spacetime. Reidel, Dordrecht. http://dx.doi.org/10.1007/978-94-009-4492-3

[16] Bacry, H. and Levy-Leblond, J.M. (1968) Journal of Mathematical Physics, 9, 1605. http://dx.doi.org/10.1063/1.1664490

[17] Santana, A.E., Matos-Neto, A. and Vianna, J.D.M. (1994) Hadronic Journal, 17, 539.

[18] Rovelli, C. (2014) Foundations of Physics, 44, 91-104. http://dx.doi.org/10.1007/s10701-013-9768-7

[19] Oliveira, M.D., Fernandes, M.C.B., Khanna, F.C., Santana, A.E. and Vianna, J.D.M. (2004) Annals of Physics, 312, 492-510. http://dx.doi.org/10.1016/j.aop.2004.03.009

[20] Brading, K. and Castellanis, E. (2003) Symmetry in Physics: Philosophical Reflections. Cambridge University Press, Cambridge.

[21] Khanna, F.C., Malbouisson, A.P.C., Malbouisson, J.M.C. and Santana, A.E. (2009) Thermal Quantum Field Theory: Algebraic Aspects and Applications. World Scientific, Singapore.

[22] Khanna, F.C., Malbouisson, A.P.C., Malbouisson, J.M.C. and Santana, A.E. (2014) Physics Reports, 539, 135-224. http://dx.doi.org/10.1016/j.physrep.2014.02.002

[23] Levy-Leblond, J.M. (1963) Journal of Mathematical Physics, 4, 776. http://dx.doi.org/10.1063/1.1724319

[24] Levy-Leblond, J.M. (1967) Communications in Mathematical Physics, 4, 157-176. http://dx.doi.org/10.1007/BF01645427

[25] Levy-Leblond, J.M. (1967) Communications in Mathematical Physics, 6, 286-311. http://dx.doi.org/10.1007/BF01646020 
[26] Ludwig, G. and Thurler, G. (2006) A New Foundation of Physical Theories. Springer, Berlin.

[27] Weinberg, S. (2011) The Quantum Theory of Fields I. Cambridge University Press, Cambridge.

[28] Peskin, M.E. and Schroeder, D.V. (1995) An Introduction to Quantum Field Theory. Addison-Wesley, New York.

[29] Toll, J.S. (1956) Physical Review, 104, 1760-1770. http://dx.doi.org/10.1103/PhysRev.104.1760

[30] Schroer, B. (2012) Foundations of Physics, 42, 1481-1522. http://dx.doi.org/10.1007/s10701-012-9676-2

[31] Plácido, H.Q., Bunchaft, R. and Santana, A.E. (1992) Hadronic Journal, 15, 225-238.

[32] Newton, I. (1995) The Principia. Prometheus Book, New York.

[33] Umezawa, H. (1993) Advanced Field Theory: Micro, Macro and Thermal Physics. American Institute of Physics, New York. 


\section{Appendix}

In this appendix we present a brief review of basic facts of non-abelian gauge theories in order to emphasize the Constitutive Elements structure. The Lagrangian of the free Dirac field describing $N$-fermions is written as

$$
\mathcal{L}_{0}(\psi, \partial \psi)=\bar{\psi}(x)[\gamma \cdot i \partial-m] \psi(x)=\sum_{i, j=1}^{n} \bar{\psi}_{i}(x)[\gamma \cdot i \partial-m] \delta_{i j} \psi_{j}(x),
$$

where $\alpha$ is the index describing the component of the spinor and the indices $i$ and $j$ stand for the $N$ types of fermions. This Lagrangian is invariant under the Poincaré group. Besides we find that $\mathcal{L}_{0}$ is also invariant under the phase transformation $\psi^{\prime}(x)=U(\lambda) \psi(x)$, where $U(\lambda)=\mathrm{e}^{i \lambda}$, with $\lambda$ being a real parameter independent of the space-time coordinates. It is important to notice that $U(\lambda)$ is unitary and as such a particular case of the $\mathrm{U}(1)$ group. In addition, this phase transformation does not affect the space-time coordinates; then the indices of the $\gamma$-matrices are invariant. Considering these characteristics of $U(\lambda)$, a generalization of this symmetry can be carried out in two directions. One is associated with the components of $\psi_{j}(x)$, since we have analyzed the phase transformation $U=\mathrm{e}^{i \lambda}$ as being the same for any type of fermions, that is for all $j=1,2, \cdots, N$. In this case, we can take $U$ as an $N \times N$ matrix in the indices $i$ and $j$. The other possible non-trivial extension is to consider $U$ as a space-time point dependent. With this characteristics, a transformation of $\psi_{j}(x)$ can be formally written as

$$
\psi_{i}^{\prime}(x)=U_{i j}(x ; \lambda) \psi_{j}(x) \text { and } \bar{\psi}_{i}(x)=\bar{\psi}_{j}(x) U_{j i}^{\dagger}(x ; \lambda),
$$

where repeated Latin indices are summed. Since this transformation $U_{i j}(x ; \lambda)$ is connected to the identity, we write

$$
U(x ; \lambda)=\mathrm{e}^{-i g \lambda(x)}=\mathrm{e}^{-i g \lambda_{r}(x) t_{r}},
$$

where $\lambda_{r}(x)$ are real functions of the space time coordinates, $g$ is a constant to fix the units and the operators $t_{j}$ are the generator of the gauge group and satisfy the Lie algebra $\left[t_{r}, t_{s}\right]=c_{r s l} t_{l}$, where $c_{r s l}$ are the structure constants of the group, with $r, s, l=1,2, \cdots, N$, where $N$ specifies the number of the group generators $t_{r}$. This number is equivalent to the maximal number of independent elements of the Lie algebra. Since we consider a finite dimensional representation for these generators, we can select the most natural one, defined by the adjoint representation. In this case, each matrix $t_{r}$ is given in the form $\left(t_{r}\right)_{s l}=\left(c_{r s l}\right)$. It is important to emphasize that the representation is also specified, in this case, by the symmetry, which in turn is given by the structure constants $c_{r s l}$.

The set of symmetries is specified by

$$
\Omega=(\Lambda, U(x ; \lambda))
$$

where $\Lambda$ is the space-time Lorentz symmetry and $U(x ; \lambda)$ describes the symmetry associated with internal degrees of freedom.

The Lagrangian $\mathcal{L}_{0}$ is no longer invariant under the transformation given by Equation (9); i.e. by $\Omega$. This invariance is, however, accomplished by including a new field, say $A$, interacting with the field $\psi$. These new terms involving $A$ have to satisfy the following conditions: (i) the Lagrangian describing the kinematic aspect of the field $A$ has to be invariant by $\Omega$; then the components of the field $A$ are in the form: $A_{i j}^{\mu}$; (ii) the field $A_{i j}^{\mu}$ is transformed under the gauge transformation, in such a way to cancel any non-invariant term in $\mathcal{L}_{0}$. This process, which can be improved step-by-step [21] [28], leads to the following gauge invariant Lagrangian

$$
\mathcal{L}=\bar{\psi}(x)\left[i D_{\mu} \gamma^{\mu}-m\right] \psi(x)-\frac{1}{4} F_{\mu \nu} F^{\mu v}
$$

where the following definitions are used. (i) The term $D_{\mu}(A)$ is a gauge invariant derivative, called covariant derivative given by

$$
D_{\mu}=\partial_{\mu}+i g A_{\mu}=I \partial_{\mu}+i g A_{\mu}^{r}(x) t^{r}
$$

(ii) Using the fact that $D_{\mu}$ is gauge invariant, the second-rank tensor field is given by 


$$
F_{\mu \nu}=D_{\mu} A_{\nu}-D_{v} A_{\mu}=\partial_{\mu} A_{\nu}-\partial_{\nu} A_{\mu}+g\left[A_{\mu}(x), A_{\nu}(x)\right] .
$$

The gauge invariance of $\mathcal{L}$ is established by defining the way that the field $A$ transforms under $U$; i.e.

$$
A_{\mu}^{\prime}(x)=U(x ; \lambda) A_{\mu}(x) U^{-1}(x ; \lambda)+\frac{i}{g} \partial_{\mu} U(x ; \lambda) U^{-1}(x ; \lambda) .
$$

For infinitesimal transformation, where $\lambda_{a}(x) \ll 1, U(x ; \lambda)$ can be written as

$$
U(x ; \lambda)=I+i g \lambda_{a}(x) l_{a} .
$$

Then we obtain the expression

$$
A_{\mu}^{\prime}(x)=A_{\mu}(x)-\partial_{\mu} \lambda(x)+i g\left[\lambda(x), A_{\mu}(x)\right],
$$

where $\lambda(x)=\lambda_{r}(x) t_{r}$. For the sake of algebraic consistency, $A_{\mu}(x)$ has to be expanded in terms of the generators of the gauge Lie algebra, i.e. $A_{\mu}(x)=A_{\mu}^{r}(x) t^{r}$, such that

$$
\left[\lambda(x), A_{\mu}(x)\right]=\left[\lambda_{r}(x) l_{r}, A_{\mu, s}(x) l_{s}\right]=\lambda_{r}(x) A_{\mu, s}(x)\left[l_{r}, l_{s}\right]=\lambda_{r}(x) A_{\mu, s}(x) c_{r s l} l_{l}
$$

In this equation, each $l_{l}$ is given in term of $N \times N$-matrix.

There is an arbitrariness in the definition of $A_{\mu}$, Equation (11), due to its $\lambda$ dependence in the gauge transformation. This can be fixed by a proper choice of $\lambda^{r}(x)$, i.e. by imposing some constraints on the field $A_{\mu}^{r}(x)$. The simplest linear form in $A_{\mu}^{r}(x)$ that be $\Omega$-invariance is given by $\partial^{\mu} A_{\mu}^{r}(x)=0$. We consider a font term for the field $A$, which is written as $J_{r}^{\mu}(x)$. These two are included in a new term in a Lorentz invariant Lagrangian, i.e.

$$
\mathcal{L}_{\text {ext }}=-\frac{1}{2 \alpha}\left(\partial^{\mu} A_{\mu}^{r}(x)\right)^{2}+A_{\mu}^{r}(x) J_{r}^{\mu}(x)
$$

The final results are independent of $\alpha$. For $\alpha=1(0)$, the gauge is called also Feynman (Landau) gauge and the Lagrangian term is called the gauge fixing. This Lagrangian is useful to establish the quantization in some cases, as the electromagnetic field. However, this is not gauge invariant in general. Such a difficulty is overcome with a proper definition of a generating functional, which are defined by using the notion of ghost fields, which is a well know procedure [28]. 
Scientific Research Publishing (SCIRP) is one of the largest Open Access journal publishers. It is currently publishing more than 200 open access, online, peer-reviewed journals covering a wide range of academic disciplines. SCIRP serves the worldwide academic communities and contributes to the progress and application of science with its publication.

Other selected journals from SCIRP are listed as below. Submit your manuscript to us via either submit@scirp.org or Online Submission Portal.
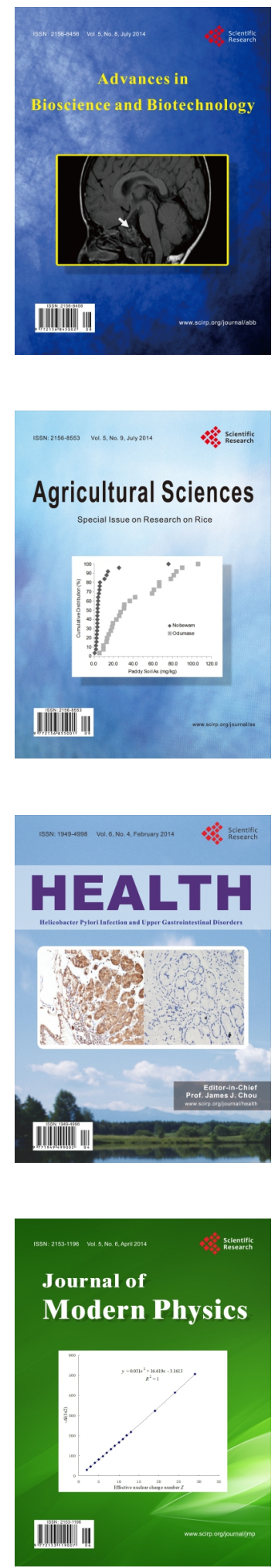
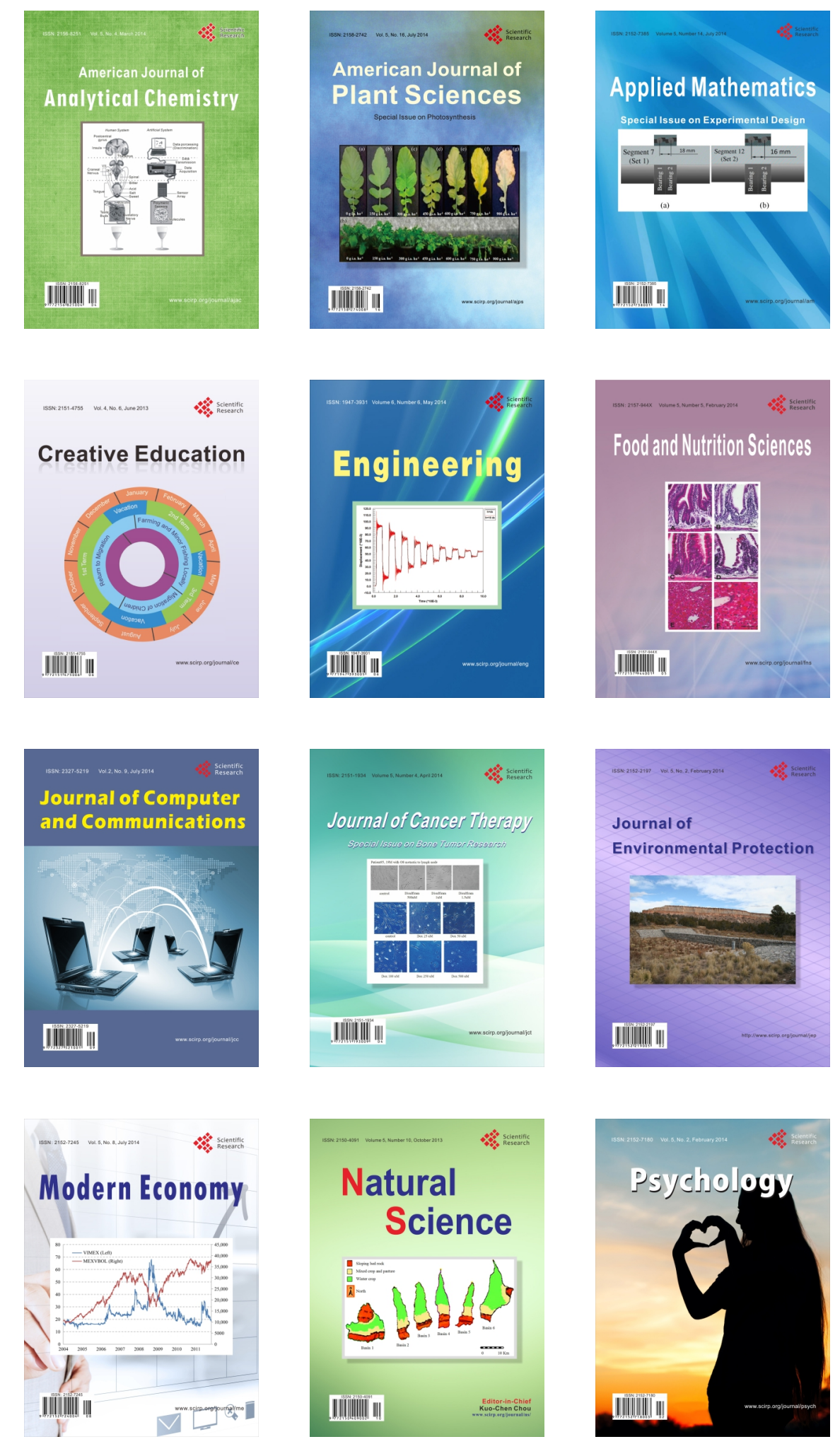